\title{
Invited commentary: Evidence-based practise and physical therapy
}

\author{
Michael Wolzt
}

Received: 30 October 2015 / Accepted: 7 December 2015 / Published online: 12 January 2016

(C) Springer-Verlag Wien 2015

Physical therapy is a well-accepted intervention by patients due to its multimodal and directly administered interventions. Unlike the rather anonymous pattern of drug prescription, where the medicines are dispensed by yet another party, subjects feel that their complaints are taken seriously by therapists: symptoms are seriously analysed, patient preferences are accounted for and treatments continuously refined during the course of a therapy. This tailored approach provides patient-centred attention and may result in a placebo-like effect of interventions in many cases.

Treatments are selected on the basis of availability and integrate expert opinion with research evidence. However, the evidence for effectiveness of many interventions in physical therapy is limited and often over-estimated, despite the frequent application of specific treatments. For example, Cochrane Reviews list $>300$ entries for "physical therapy" [1]. Among these systematic reviews, $<40$ publications analyse interventions to treat low back pain such as back schools, massage, various exercises and other typical methods.

The strength of recommendations from systematic reviews is based on the quality of original reports. Most studies which assess the application of physical therapy are limited by the heterogeneity of populations, interventions, sample size, lack of appropriate controls and treatment assignments, and robustness of outcome parameters. Low-level laser therapy (LLLT) can be used to treat musculoskeletal disorders such as non-specific low back pain but there is insufficient data to draw firm conclusions on the clinical effect [2]. Methodologically rigorous randomized

\section{Wolzt $(\bowtie)$}

Department of Clinical Pharmacology, Medical University of Vienna,

Waehringer Guertel 18-20,

1090 Vienna, Austria

e-mail: michael.wolzt@meduniwien.ac.at controlled trials are required to evaluate the effects of LLLT compared to other treatments, different lengths of treatment, wavelengths and dosages.

One of the reasons for the obvious lack of evidence is the fact that the wide range of treatment modalities is not analysed for its clinical benefit in a standardized and structured way. A generally accepted assessment tool is not established. Second, many physical therapies use medical devices. These devices can be operated in European Union Member States if they carry a CE mark, which is awarded by a number of notified bodies in Europe upon application and is not issued directly by national competent authorities after review of clinical safety and efficacy data. Further, the apparent public underreporting of experiences prevents integration of evidence into a broader discussion or (inter-) national benchmarking of data.

Our current expectation that expert knowledge and skilful hands deliver the best of possible care in physical therapy for our conditions is not falsified. On the other hand, it is time to put the various available treatments under close scrutiny to identify best standard of care. The consumers and payers of a healthcare system deserve a diligent review of the costs and benefits of a presently rather befogged scenario of therapies many of which are device-based.

\section{Conflict of interest}

The author declares that there are no actual or potential conflicts of interest in relation to this article.

\section{References}

1. The Cochrane Database of Systematic Reviews. http:// www.cochranelibrary.com/. Accessed: 15. Dec. 2015.

2. Yousefi-Nooraie R, Schonstein E, Heidari K, Rashidian A, Pennick V, Akbari-Kamrani M, Irani S, Shakiba B, Mortaz Hejri SA, Mortaz Hejri SO, Jonaidi A. Low level laser therapy for nonspecific low-back pain. Cochrane Database Syst Rev. 2008 (2):CD005107. doi:10.1002/14651858.CD005107. pub4. 